\title{
Electrochemical Sensing of Biotin Using Nafion-Modified Boron- Doped Diamond Electrode
}

\author{
Alyah Buzid, ${ }^{\dagger, \ddagger}$ Gerard P. McGlacken, ${ }^{\ddagger}$ Jeremy D. Glennon, ${ }^{\dagger \neq}$ and John H. T. Luong $*,+\neq 0$ \\ ${ }^{\dagger}$ Innovative Chromatography Group, Irish Separation Science Cluster (ISSC) Ireland, University College Cork, Western Road, Cork \\ T12 YN60, Ireland \\ ${ }^{\ddagger}$ School of Chemistry and Analytical \& Biological Chemistry Research Facility (ABCRF), University College Cork, College Road, \\ Cork T12 YN60, Ireland
}

\section{Supporting Information}

ABSTRACT: Nafion formed on the surface of a boron-doped diamond electrode allows for a chemosensing system for biotin. The modified electrode is capable of oxidizing biotin and offers a detection limit of $5 \mathrm{nM}$, the average normal level of biotin in blood plasma. The developed method was successfully applied to determine biotin in human plasma samples and a popular health product as two popular models.

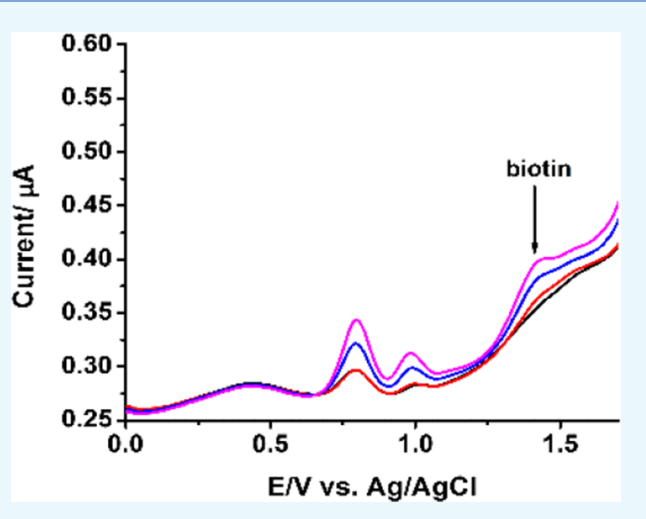

\section{INTRODUCTION}

For the most part, the use of biotin (also known as coenzyme $\mathrm{R}$, vitamin $\mathrm{H}$ or $\mathrm{B} 7$ ) is limited to labeling studies of biomolecules for diversified applications. ${ }^{1}$ As a stable and small molecule (MW of $244.3 \mathrm{Da}$ ), biotin rarely alters the function of resulting conjugated molecules. Biotin acts as a catalyst for carboxylase enzymes. ${ }^{2}$ The adequate intake of biotin for adults is $30-100 \mu \mathrm{g} /$ day based on as estimated from Food and Nutrition Board ${ }^{3}$ for health benefits such as stimulating hair growth and treating certain medical conditions including biotin deficiency (BTD), lipid disorders, diabetes, and diabetic peripheral neuropathy. ${ }^{4-7}$ However, supraphysiological consumption of biotin in a plethora of health products results in its high concentration in blood. Free biotin in patient samples is posing a serious threat to immunodiagnostics, as evident by the recent safety alert by the US Food and Drug Administration (FDA). ${ }^{8}$ Free biotin can interfere with the diagnostic results of assays employing biotin-avidin interactions, by competing with the biotinylated recognition element, thereby leading to false positives or false negatives. ${ }^{9}$ Thus, a rapid and inexpensive method for the determination of biotin in patient samples is urgently needed. The level of biotin might be used to correct for its interference in immunoassays using the avidin-biotin chemistry. The current popular method is based on 4-hydroxyazobenzene-2-carboxylic acid which forms a reversible yellow-orange color complex with avidin. ${ }^{10}$ This avidin-binding dye is stoichiometrically displaced by biotin, leading to the subsequent absorption change, which provides a basis for the assay.
There are only a few studies pertaining to direct electroanalysis of biotin. Pioneering work, dating back over 40 years, unravels an irreversible one-electron reduction of biotin at $-1.8 \mathrm{~V}$, in dimethylformamide (DMF)-water solutions. ${ }^{11,12}$ The topic was revisited by Lauw et al. to investigate biotin reduction at a platinum (Pt) surface, in DMF and dimethylsulfoxide (DMSO) supported by the organic salt tetrabutylammonium hexafluorophosphate (TBAPF). ${ }^{13}$ Therein, biotin at $2 \mathrm{mM}$ is reduced by one-electron to form a carboxylate anion and dihydrogen in DMF and DMSO. ${ }^{13}$ This behavior is more pronounced in DMF than DMSO with a $\mathrm{Pt}$ electrode poised at $-1.6 \mathrm{~V}$ versus $\mathrm{Fc} / \mathrm{Fc}^{+}(\mathrm{Fc}=$ Ferrocene $)$ but does not occur if glassy carbon is used as the electrode material. ${ }^{13}$ This approach is not applicable for the assay of biotin in aqueous samples because of the severe interference of water, even in minute quantity, particularly at high positive or negative potentials, as discussed later.

In general, biotin in foodstuffs and biological fluids can be analyzed by bioassays, avidin-streptavidin-binding assays, or fluorescent derivative assays. ${ }^{14}$ However, biotin metabolites and analogs interfere with the assay, particularly for the avidin assay as addressed later. For a specific analysis of biotin in the presence of its metabolites, the sample must be subjected to high-performance liquid chromatography-mass spectroscopy (HPLC-MS) followed by avidin-streptavidin-binding assays. ${ }^{15}$ The former procedure is capable of separating biotin

Received: June 1, 2018

Accepted: June 28, 2018

Published: July 12, 2018 

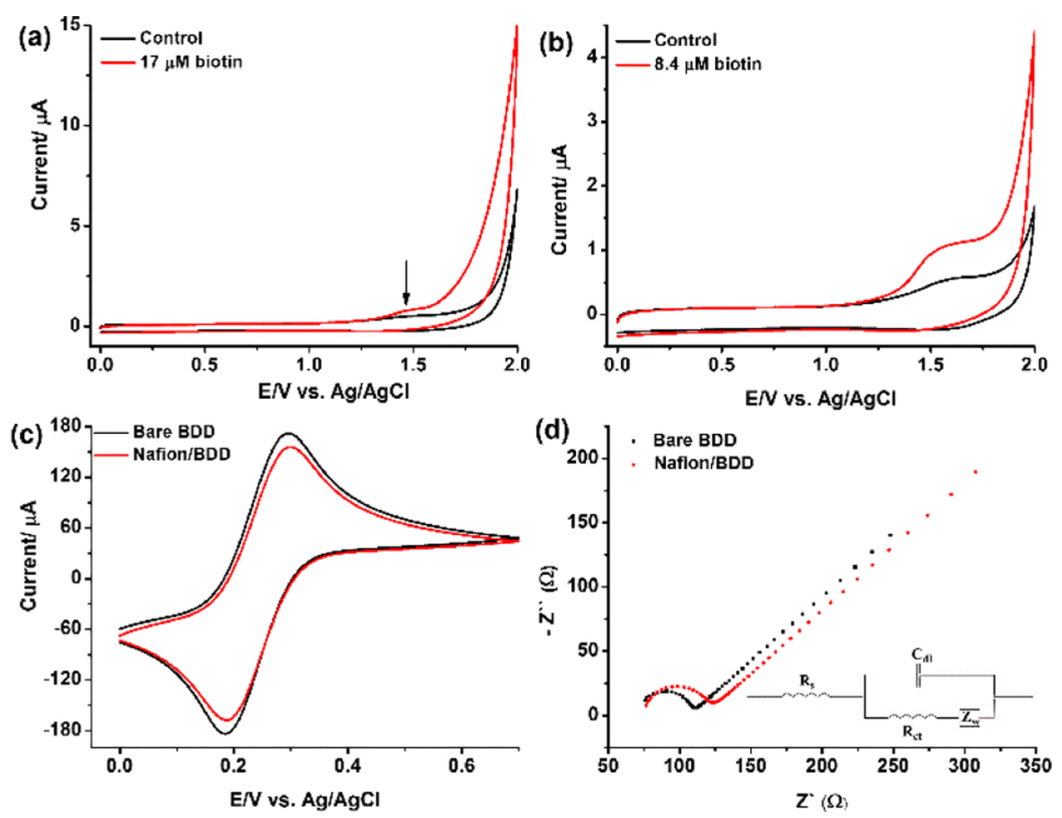

Figure 1. CVs of biotin obtained by the Nafion-modified BDD electrode vs $\mathrm{Ag} / \mathrm{AgCl}$ using (a) $0.2 \mathrm{M}$ acetate buffer, $\mathrm{pH} 4$; (b) $0.2 \mathrm{M}$ phosphate buffer, $\mathrm{pH} \mathrm{2,} \mathrm{a} \mathrm{scan} \mathrm{rate} \mathrm{of} 100 \mathrm{mV} \mathrm{s}^{-1}$; (c) the CV of the bare and Nafion-modified BDD electrodes at a scan rate of $50 \mathrm{mV} \mathrm{s}$ and (d) EIS of the bare and Nafion-modified BDD electrodes (inset: the modified Randles circuit). Fe $(\mathrm{CN})_{6}^{4-/ 3-}(10 \mathrm{mM})$ prepared in $0.1 \mathrm{M} \mathrm{KCl}$.

from its metabolites whereas the latter detects biotin as well as its metabolites.

The boron-doped diamond (BDD) electrode has been advocated here because of its low background current, resistance to fouling, and broad potential window. ${ }^{16}$ Recent reports have demonstrated its applicability for detection of Pseudomonas quinolone signal, 2-heptyl-4-hydroxyquinoline, pyocyanin, ${ }^{17-19}$ 2-(2-hydroxyphenyl)-thiazole-4-carbaldehyde (IQS), ${ }^{20}$ and barakacin $^{21}$ as a molecular signature of Pseudomonas aeruginosa. There are a number of analytical techniques reported for biotin determination such as spectrometry, ${ }^{22}$ polarography, ${ }^{12}$ thin-layer chromatography, ${ }^{23}$ capillary electrophoresis, ${ }^{24}$ and high-performance liquid chromatography. ${ }^{25-27}$

This communication unravels a new strategy for specific analysis of biotin based on its electrochemical oxidation of biotin at the surface of a Nafion-modified BDD electrode in an aqueous media. Two popular model systems: a natural product and human blood plasma will be tested to validate the proof of concept. In our opinion, there is no report which exploits such features for the electroanalysis of biotin.

\section{RESULTS AND DISCUSSION}

Behavior of the Bare BDD Electrode. Considering the limited aqueous solubility of biotin $(0.2 \mathrm{mg} / \mathrm{mL}),{ }^{28}$ the experiment was first conducted using a bare BDD electrode in acetonitrile $(\mathrm{MeCN})$ supported by TBAPF. This organic salt is highly soluble in $\mathrm{MeCN}$ and offers a very low current background over a wide potential range. Furthermore, the high miscibility of $\mathrm{MeCN}$ with water (40:60\%, v/v) accommodates the analysis of aqueous samples. In summary, both cyclic voltammetry $(\mathrm{CV})$ and amperometry $i-t$ curve confirmed the oxidation of biotin (also prepared in $\mathrm{MeCN}-\mathrm{TBAPF}$ ) at the applied potential of $+1.8 \mathrm{~V}$ with a detection limit (LOD) of 3 $\mu \mathrm{M}$ (Figure $\mathrm{S} 1 \mathrm{a}, \mathrm{b})$. In contrast, there was significant interference using aqueous biotin samples because of peaks derived from oxygen evolution and solvent breakdown ${ }^{29}$
(Figure S1c). When the MeCN-TBAPF system was applied to an aqueous biotin sample, the above LOD was no longer attained. Even at water: electrolyte volume ratios below 1:50, the adsorbed water onto the electrode $\left(\mathrm{BDD}-\mathrm{H}_{2} \mathrm{O}\right)$ was oxidized at high potentials $\left(2 \mathrm{H}^{+}+2 \mathrm{e}^{-}+1 / 2 \mathrm{O}_{2}\right)$, as reflected by a current that increased steadily at potentials beyond +1.8 V. This phenomenon of oxygen evolution via water hydrolysis is well-known ${ }^{30}$ and limits the applied potential of most metallic electrodes to below $0.8-0.9 \mathrm{~V}$.

In $\mathrm{MeCN}$, the released protons can exit into the reaction/ diffusion layer near the electrode surface and can be accommodated by $\mathrm{MeCN}$ as a proton acceptor. Solvent ( $\mathrm{MeCN}$ and TBAPF) breakdown could also occur here, resulting in further interference peaks. Indeed, the solvent breakdown has been known at the Pt surface in a $\mathrm{MeCN} /$ tetra$n$-butylammonium tetrafluoroborate system, ${ }^{29}$ whereas $\mathrm{BF}_{4}{ }^{-}$ acts as a fluorinating agent in $\mathrm{MeCN}$ at $+2.0 \mathrm{~V}$ together with the formation of $\mathrm{HF}^{3}$

In this work, an attempt using a high negative potential for the reduction of biotin was also not successful in the presence of a small amount of water from the biotin sample at the BDD electrode in MeCN-TBAPF. The CV exhibited a very high and broad emerging peak at $-1.8 \mathrm{~V}$ and a small peak at $-2 \mathrm{~V}$, which overlapped with the biotin reduction peak (Figure S2). These two peaks could be attributed to the reduction of water adsorbed on $\mathrm{BDD}\left(\mathrm{H}_{2} \mathrm{O}+\mathrm{e}^{-} \rightarrow 1 / 2 \mathrm{H}_{2}\right.$ (gas) $\left.+\mathrm{OH}^{-}\right)$. The formed $\mathrm{OH}^{-}$has been known to react with $\mathrm{MeCN}$ to form acetamide $\left(\mathrm{CH}_{3} \mathrm{CO}-\mathrm{NH}_{2}\right)$, resulting in the solvent breakdown as reported by Pons and Khoo ${ }^{32}$ which again could lead to interfering peaks. Evidence pointing to acetamide formation was provided by the presence of an IR peak at $1650 \mathrm{~cm}^{-1}$ on an electrolyte sample taken after 30 cycles of voltammetry $\left(-2.5\right.$ to $+2.5 \mathrm{~V}$ at $100 \mathrm{mV} \mathrm{s}^{-1}$ ), (Figure S3). The $\mathrm{OH}^{-}$could also be formed from the reduction of dissolved oxygen, involving two different pathways. Oxygen is reduced directly to $\mathrm{OH}^{-}$by a four-electron transfer, via $\mathrm{H}-\mathrm{O}_{2}$ as an intermediate species, followed by two further electron-transfer processes to 
yield $4 \times \mathrm{OH}^{-} \cdot 33$ Alternatively, the hydride ion $\left(\mathrm{H}^{-}\right)$in the solution is known to deprotonate $\mathrm{MeCN}$ to $\mathrm{CH}_{2}-\mathrm{CN}$, which then attacks another $\mathrm{MeCN}$ to form a dimer. ${ }^{29}$ Besides the restriction of water, the $\mathrm{MeCN}-\mathrm{TBAF}$ approach lacks the detection sensitivity required for blood screening for biotin, ranging from $35 \mathrm{nM}$ to $2.02 \mu \mathrm{M}$ after single doses of $1 \mathrm{mg}$ or 100 mg. $^{9}$

Performance of the Nafion Modified BDD Electrode. The experiments were then performed in aqueous electrolytes using $0.2 \mathrm{M}$ phosphate buffers at $\mathrm{pH} 4$ and $\mathrm{pH}$ 2. The Nafion was drop-casted on the BDD electrode. A representative $\mathrm{CV}$ of the Nafion-modified electrode with and without biotin is shown in Figure 1a,b, which shows the emerging oxidation peak of the biotin oxidation product(s). It is important to mention that the band around $+1.5 \mathrm{~V}$ is due to the phosphate buffer in Figure $1 \mathrm{~b}$, whereas the acetate buffer does not induce any band (Figure 1a). The polymeric structure of Nafion contains a hydrophobic $\left(\mathrm{CF}_{2}-\mathrm{CF}_{2}\right)$ backbone, along with two oxygen atoms and a hydrophilic sulfonic acid $\left(\mathrm{SO}_{3}^{-}\right)$group, which contributes to its electron-withdrawing effect. Thus, Nafion displays both hydrophobic and ionic interactions with the BDD surface to form a stable layer, as confirmed by $\mathrm{CV}$ and electrochemical impedance spectroscopy (EIS) measurements (Figure 1c,d). The parameters obtained for the bare and Nafion-modified BDD electrodes using an equivalent circuit $R_{\mathrm{s}}$ $\left(C_{\mathrm{dl}}\left(R_{\mathrm{ct}} Z_{\mathrm{w}}\right)\right)$ is shown in Table $S 1$. The Nafion-modified BDD electrode is reusable and required to be cleaned only in $0.5 \mathrm{M}$ $\mathrm{H}_{2} \mathrm{SO}_{4}$. The electrode was tested using the ferri/ferrocyanide redox couple as described in Figure 1c,d. The BDD itself is prepared by chemical vapor deposition with hydrogen gas and thus is recognized to be hydrogen-terminated. ${ }^{16}$

A simple $i-t$ curve poised at $+1.8 \mathrm{~V}$ shown in Figure $2 \mathrm{a}$ illustrated an LOD of $<1 \mu \mathrm{M}$ for biotin for the Nafion-
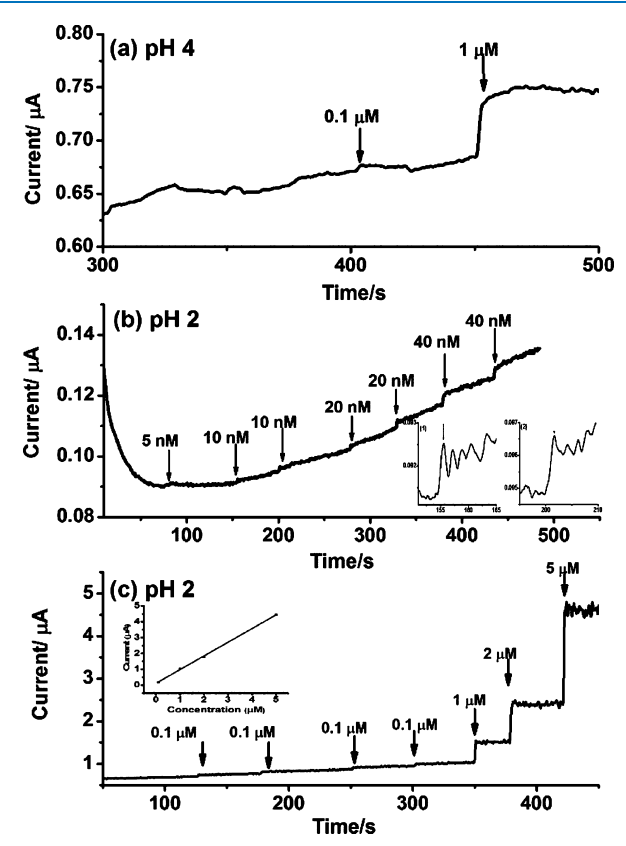

Figure 2. (a) $i-t$ curve for LOD determination using $0.2 \mathrm{M}$ acetate buffer, $\mathrm{pH}$ 4. (b) $i-t$ curve for LOD determination using $0.2 \mathrm{M}$ phosphate buffer, $\mathrm{pH} 2$; $10 \mathrm{nM}$ biotin [(b), insert]. (c) Calibration curve of biotin. The detection was achieved on the Nafion-modified $\mathrm{BDD}$ electrode vs $\mathrm{Ag} / \mathrm{AgCl}$, the potential was at $+1.8 \mathrm{~V}$, using $0.2 \mathrm{M}$ phosphate buffer, $\mathrm{pH} 2$. modified BDD electrode at $\mathrm{pH}$ 4. Remarkably, an estimated LOD of $5 \mathrm{nM}$ of biotin was obtained at $\mathrm{pH} \mathrm{2,} \mathrm{when} \mathrm{the}$ Nafion-modified electrode was poised at $+1.8 \mathrm{~V}$ (Figure $2 \mathrm{~b}$ ) because of the lower and stable background current. Figure $2 c$ shows the performance of the Nafion-modified BDD electrode at high concentrations of biotin, and the calibration curve of biotin exhibited excellent linearity $\left(R^{2}>0.99\right)$ (Figure $2 \mathrm{c}$ insert).

A premixed biotin-avidin or biotin-streptavidin sample provoked no signal response (Figure $3 \mathrm{a}, \mathrm{b}$ ). Conversely, the
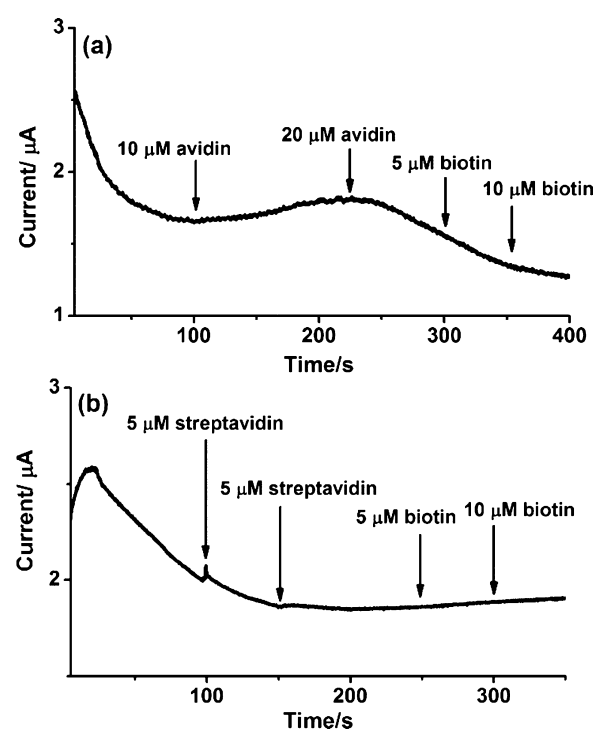

Figure 3. (a) $i-t$ curve obtained following the sequential addition of avidin and biotin. (b) $i-t$ curve obtained following the sequential addition of streptavidin and biotin. The detection was achieved on the Nafion-modified $\mathrm{BDD}$ electrode vs $\mathrm{Ag} / \mathrm{AgCl}$, the potential was at $+1.8 \mathrm{~V}$.

response signal of biotin decreased significantly when the protein (avidin or streptavidin) was introduced to the electrochemical cell, illustrating the formation of the biotinprotein complex. It is well-known that avidin and streptavidin possesses a hydrophobic cavity that binds four biotin molecules irreversibly. ${ }^{34}$ There are many interesting works on the oxidation and reduction of biotin. The oxidation product of biotin by electrochemical oxidation is not known and requires further investigation. The sulfur of biotin can be oxidized by $\mathrm{H}_{2} \mathrm{O}_{2}$, oxone, and other strong oxidants to form biotin sulfoxide ${ }^{35}$ and biotin sulfone. ${ }^{36}$ However, experimentally, such reactions must be performed for several hours, usually at elevated temperature. Using a strong reductant (Raney nickel) was reported to reduce biotin to desthiobiotin. ${ }^{37}$ In a contrasting report, biotin is not activated by Raney nickel and the tetrahydrofuran moiety of oxybiotin appears quite robust. ${ }^{38}$ Bacterial degradation of biotin using a $\beta$ oxidative system $^{39}$ drives oxidation of the valeric side chain, before hydrolysis of the ureido ring to form bisnorbiotin and teranorbiotin. Oxybiotin, an analog of biotin (O instead of S), can be prepared from biotin by oxidation. ${ }^{40}$ In electrochemical reduction, biotin is simply reduced by one-electron to carboxylate anion and dihydrogen via a discharge of the carboxylic acid at the Pt surface. ${ }^{13}$ In this context, it is difficult to specify the oxidation product(s) produced by the modified electrode protocol described herein. As mentioned earlier, Nafion consists of a hydrophobic backbone and a hydrophilic 
sulfonic acid group. When the water content of the membrane is increased, the proton simultaneously diffuses through a polymer electrolyte membrane, and the Nafion membrane is dehydrated due to the electroosmotic drag from hydronium ion. ${ }^{41}$ Also, the rate of proton transport depends on the water concentration. ${ }^{42}$ Thus, the initial step of electrochemical oxidation using the Nafion-modified BDD electrode involves the partial protonation of biotin at the sulfur of the tetrahydrothiophene ring and possibly the $\mathrm{C}=\mathrm{O}$ of the ureido group. Then, electrochemical oxidation of protonated biotin results $^{3}$ in a distinct possibility that the oxidation products involves sulfoxide or sulfone groups, ${ }^{43,44}$ or even oxybiotin ${ }^{40}$ as shown in Scheme 1. It should be noted that the oxidation

Scheme 1. Postulated Electrochemical Oxidation of Biotin To Form Biotin Sulfone (1) or Biotin Sulfoxide (2) or Oxybiotin (3)
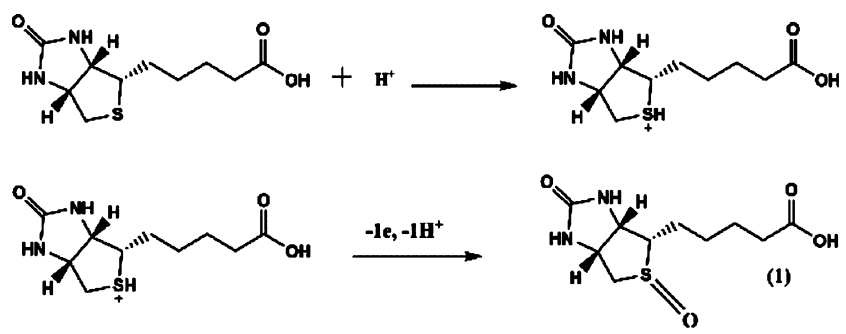

OR
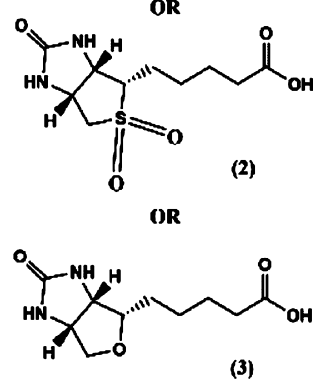

(3)

capability of the BDD system also leads to the possibility of accessing biotin analogs with different biological activities using electrochemical means and without recourse to atomuneconomic oxidizing agents.

Analytical Performance of the Chemosensor. The chemosensing approach was then applied to the analysis of a commercial health product with a high dose of biotin $(900 \mu \mathrm{g})$ together with other components. A simple measurement by the $i-t$ curve was sufficient to estimate biotin content, based on the signal response invoked (Figure 4). A single biotin tablet $(900 \mu \mathrm{g})$ was dissolved in deionized water. The quantity of biotin was estimated to be between 874 and $884 \mu \mathrm{g}$, compared with $900 \mu \mathrm{g}$ as specified by the manufacturer.

Immunoassays for biotin have been commercially available using a microtiter plate coated with avidin or streptavidin. In brief, the enzyme-labeled biotin and the biotin-containing sample are added, which compete with each other for the avidin binding sites. After the removal of unbound enzymelabeled biotin, a specific enzyme substrate is added to trigger color change with a resulting absorbance change proportional to the biotin concentration of the sample. However, the assay is not specific for biotin because some known biotin analogs also bind to the coating avidin-streptavidin.

The cross-reactivity is about $83 \%$ for biocytin $34.7 \%$ for biotin- $d$-sulfoxide, $28.2 \%$ for biotin sulfone, $24.5 \%$ for biotin- $l$ -

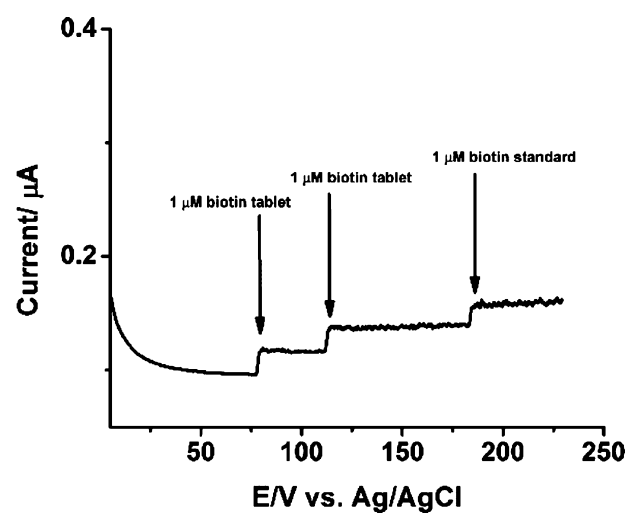

Figure 4. $i-t$ curve of $1 \mu \mathrm{M}$ biotin tablet and $1 \mu \mathrm{M}$ of the biotin standard. The detection was achieved on the Nafion-modified BDD electrode: $+1.8 \mathrm{~V}$ vs $\mathrm{Ag} / \mathrm{AgCl}$, using $0.2 \mathrm{M}$ phosphate buffer, $\mathrm{pH} 2$.

sulfoxide, and $5.5 \%$ compared with $100 \%$ for biotin. ${ }^{45}$ This method is referred as the total avidin binding assay, that is, biotin together with its analogs. Nevertheless, the assay should be applicable for the analysis of biotin in food samples considering the absence of such biotin analogs. However, the biotin analogs are not negligible but account for half of the total avidin-binding substances in human plasma. ${ }^{46}$ This critical issue has pointed out who require hemodialysis from the analysis of patients' blood for biotins. ${ }^{47}$ Of notice is the specific detection of biotin by the Nafion-modified BDD electrode because the above biotin analogs are not oxidized at this applied potential for the oxidation of biotin. Apparently, the difference between the results obtained by the electrode and the immunoassay reflects the level of the biotin analogs. This finding leads to a significance application considering the simplicity of rapidity of electrochemical analysis over HPLC for separation of biotin from its metabolites.

The detection of biotin from blood and blood plasma serves as an indicator of BTD and in some cases it is important for the patients who are subjected to hemodialysis or under biotin therapy with high dosage. Therefore, the modified electrode was then applied to the plasma sample, a more challenging task because of the presence of endogenous electroactive compounds. Indeed, numerous human blood components have been identified in this very complex matrix. ${ }^{48}$ The commercial plasma sample exhibited several major differential pulse voltammetry (DPV) peaks, illustrating the presence of endogenous electroactive molecules including uric acid, dopamine, and so forth. (Figure 5). Indeed, numerous human blood components have been identified in this very complex matrix. ${ }^{48}$ The two peaks obtained from plasma samples were identified as dopamine and tryptophan (Figure $5 B, C)$. Thus, the analysis of biotin in blood plasma samples could not be realized by the simple $i-t$ approach (Figure S4). The plasma samples spiked with uric acid results in an increased baseline signal (Figure S5), whereas the plasma samples spiked with tyrosine resulted in a decreased signal (Figure S6). Nevertheless, DPV enabled the detection of spiked biotin in blood plasma samples in the presence of the matrix interferences. However, colorimetric assays including Elisa using the avidin-biotin chemistry will detect biotin and biotin analogs, whereas our approach only detects biotin. Therefore, the results of the two procedures are not comparable, considering that the total metabolite concentration is about $50 \%$ that of biotin. Of course, LC-MS, an 


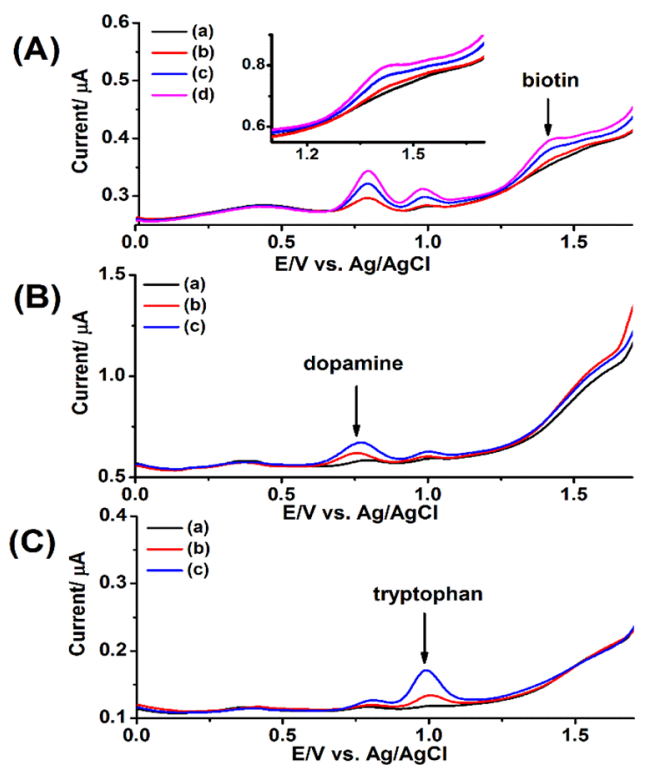

Figure 5. (A) DPVs obtained of a blank and spiked plasma sample with biotin standard. (a) $100 \mu \mathrm{L}$ blank plasma; (b) $100 \mu \mathrm{L}(2 \mu \mathrm{M})$ biotin-spiked plasma; (c) $200 \mu \mathrm{L}(4 \mu \mathrm{M})$ biotin-spiked plasma; and (d) $300 \mu \mathrm{L}(6 \mu \mathrm{M})$ biotin-spiked plasma. (B) DPVs obtained of a blank and spiked plasma sample with dopamine standard. (a) $200 \mu \mathrm{L}$ blank plasma; (b) $200 \mu \mathrm{L}$ (4 $\mu \mathrm{M})$ dopamine-spiked plasma; and (c) $400 \mu \mathrm{L}(8 \mu \mathrm{M})$ dopamine-spiked plasma. (C) DPVs obtained of a blank and spiked plasma sample with tryptophan standard. (a) $200 \mu \mathrm{L}$ blank plasma; (b) $200 \mu \mathrm{L}(4 \mu \mathrm{M})$ tryptophan-spiked plasma; and (c) $400 \mu \mathrm{L}(8 \mu \mathrm{M})$ tryptophan-spiked plasma. The detection was achieved on the Nafion-modified BDD electrode using $0.2 \mathrm{M}$ phosphate buffer, $\mathrm{pH} 2$.

expensive lab-based laboratory, will be used to compare the results obtained by the electrochemical sensor. This task is underway in our laboratories to monitor the biotin levels in patients' blood, a separate topic in our future endeavor.

The detection selectivity and sensitivity of the Nafionmodified BDD electrode deserves a brief comment here with respect to the detection limit required in clinical chemistry. Biotin plays an important role in the human organs, which might serve as a BTD index. The clinical analysis of biotin will involve over 50-60 patients from three different groups: male, female, and young people because their respective biotin levels are noticeably different and the blood level after the digestion of biotin varies significantly. Of particular interest is the beneficial effects of high-dose biotin therapy in progressive multiple sclerosis (MS), the most common disabling neurological disease in young adults. Under biotin therapy, the biotin concentration of an MS patient can reach $2.84 \mu \mathrm{M}^{49}$ A liquid chromatography-tandem mass spectrometry method (LCMS-MS) has been proven useful with a measuring range of $20 \mathrm{nM}$ to $12.3 \mu \mathrm{M}$. The electroanalysis with a detection limit of micrometer could be useful to follow the biotin level of MS patients under biotin therapy with high dosage. The sample can be preconcentrated by solid-phase extraction, a simple and fast step in analytical and clinical chemistry.

\section{CONCLUSIONS}

Nafion formed a stable layer on the surface of a BDD electrode and allowed for sensitive detection of biotin. In the absence of Nafion, the nonaqueous approach using $\mathrm{MeCN}$ and a conducting salt gave a poor detection limit. The BDD electrode displayed a wide, usable potential window at $\mathrm{pH} 2$ and was capable of oxidizing biotin, resulting in a detection limit of $5 \mathrm{nM}$. This inexpensive and rapid method is of interest for the analysis of biotin in various clinical, biological, and food samples. As described previously, the high level of biotin interferes with immunoassays based on the biotinylated molecules, resulting in falsely increased or decreased test results. This is a serious pending issue in the clinical diagnosis of biomarkers and diversified important analytes. Together with solid-phase extraction, this approach can deliver the detection sensitivity as required in clinical chemistry.

\section{EXPERIMENTAL SECTION}

Chemicals. Sodium phosphate monobasic, phosphoric acid, acetic acid, sodium acetate, biotin, potassium chloride, Nafion perfluorinated resin $(5 \%)$ in a mixture of lower aliphatic alcohols, TBAPF, potassium hexacyanoferrate, ethanol, acetonitrile $(\mathrm{MeCN})$, methanol $\left(\mathrm{CH}_{3} \mathrm{OH}\right)$, plasma from human, uric acid, tyrosine, dopamine, tryptophan, and avidin from egg white were purchased from Sigma-Aldrich (Dublin, Ireland), whereas streptavidin was purchased from Thermo Fisher Scientific (Dublin, Ireland). Deionized water (Millipore, Ireland) was utilized throughout the experiments, and all chemicals were of the analytical grade. The biotin tablet was obtained from a local pharmacy, marketed as Boots (Nottingham, England): Beauty from within, biotin (Cork, Ireland), containing $900 \mu \mathrm{g}$ biotin, in addition to dicalcium phosphate, cellulose, hydroxypropylmethyl cellulose, hydroxypropyl cellulose, silicon dioxide, talc, magnesium stearate, stearic acid, potassium aluminum silicate, and colors (titanium dioxide, indigo carmine, and carmine). Human plasma was prepared in deionized water. Then, the plasma was spiked with $100 \mu \mathrm{M}$ biotin standard and incubated for $15 \mathrm{~min}$. A blank plasma sample $(100 \mu \mathrm{L})$ was added to the electrochemical cell

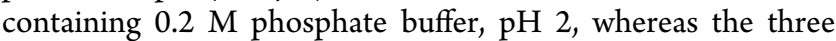
different volumes of the biotin-spiked plasma sample (100, 200 , and $300 \mu \mathrm{L}$ ) was added to the electrochemixzcal cell containing $0.2 \mathrm{M}$ phosphate buffer, $\mathrm{pH} 2$. To identify the electroactive molecules in plasma samples, the plasma was spiked with $100 \mu \mathrm{M}$ uric acid, tyrosine, dopamine, and tryptophan standard each.

Apparatus and Measurements. EIS measurement was performed at room temperature using a CHI660C electrochemical workstation ( $\mathrm{CH}$ Instrument, Austin, TX). DPV, amperometric measurement $i-t$ curve, and $\mathrm{CV}$ were used during electrochemical measurements at ambient temperature using a CHI1040A electrochemical workstation ( $\mathrm{CH}$ Instrument, Austin, TX). The electrochemical cell consists of the BDD working electrode (Windsor Scientific, Slough Berkshire, UK), a Pt wire counter electrode (Sigma-Aldrich, Dublin, Ireland), and a silver chloride $(\mathrm{Ag} / \mathrm{AgCl} / 3 \mathrm{M} \mathrm{KCl})$ reference electrode (BASi Analytical Instruments, West Layette, IN). The CV and ESI measurements were performed in $10 \mathrm{mM}$ $\mathrm{K}_{3} \mathrm{Fe}(\mathrm{CN})_{6}^{4-/ 3-}$ supported by $0.1 \mathrm{M} \mathrm{KCl}$. EIS was performed with $\mathrm{AC}$ frequency ranged between $1 \mathrm{~Hz}$ and $100 \mathrm{kHz}$ at +0.2 V.

Preparation of the Modified Electrodes. The BDD electrode was polished using 0.3 and $0.05 \mu \mathrm{m}$ alumina slurry with wet Nylon and MasterTex papers, respectively, followed by sonication in $\mathrm{MeCN}$ for $5 \mathrm{~min}$ and deionized water for 10 min. The Nafion-modified BDD electrode was obtained by drop-casting $2 \mu \mathrm{L}$ of Nafion solution $(2 \mathrm{w} / \mathrm{v} \%$, prepared in 
ethanol) on the polished BDD electrode followed by vacuum drying.

\section{ASSOCIATED CONTENT}

\section{S Supporting Information}

The Supporting Information is available free of charge on the ACS Publications website at DOI: 10.1021/acsomega.8b01209.

Cyclic voltammogram (CV), limit of detection (LOD) of biotin, and water interference of the bare BDD electrode in the TBAPF-MeCN electrolyte; $\mathrm{CV}$ of biotin reduction in the TBAPF-MeCN electrolyte on the bare BDD electrode; IR spectra of 0.2 M TBAPF in $\mathrm{MeCN}$ contains $1 \%$ water after 30 cycles of voltammetry; parameters obtained for the bare and Nafion-modified BDD electrodes using an equivalent circuit $R_{\mathrm{s}}\left(C_{\mathrm{dl}}\left(R_{\mathrm{ct}} Z_{\mathrm{w}}\right)\right)$; $i-t$ curve of the blood plasma and the biotin-spiked blood plasma; differential pulse voltammograms (DPVs) obtained for a blank and a spiked plasma sample with a uric acid standard; and DPVs obtained of a blank and a spiked plasma sample with a tyrosine standard (PDF)

\section{AUTHOR INFORMATION}

\section{Corresponding Author}

*E-mail: j.luong@ucc.ie.

\section{ORCID}

John H. T. Luong: 0000-0002-1709-1223

\section{Funding}

This research was financially supported by SFI/EI Technology Innovation Development Award (TIDA) (SFI/12/TIDA/ B2405). GPM acknowledges the support from Enterprise Ireland (CF-2017-0757-P). J.D.G. thanks the Science Foundation Ireland (08/SRC/B1412) for research funding of the Irish Separation Science Cluster (ISSC) under the Strategic Research Cluster Programme. J.D.G. and A.B. acknowledge supports by Enterprise Ireland (CF-2017-0759P). G.P.M. acknowledges supports by the Science Foundation Ireland (SFI/12/IP/1315, SFI/09/RFP/CHS2353, and SSPC2 12/RC/2275), the Irish Research Council (GOIPG/ 2013/336), and UCC for a Strategic Research Fund Ph.D. Studentship.

\section{Notes}

The authors declare no competing financial interest.

\section{REFERENCES}

(1) Szychowski, J.; Mahdavi, A.; Hodas, J. J. L.; Bagert, J. D.; Ngo, J. T.; Landgraf, P.; Dieterich, D. C.; Schuman, E. M.; Tirrell, D. A. Cleavable biotin probes for labeling of biomolecules via azide-alkyne cycloaddition. J. Am. Chem. Soc. 2010, 132, 18351-18360.

(2) McMahon, R. J. Biotin in metabolism and molecular biology. Annu. Rev. Nutr. 2002, 22, 221-239.

(3) Institute of Medicine Standing Committee. Scientific Evaluation of Dietary Reference Intakes. Dietary Reference Intakes for Thiamin, Riboflavin, Niacin, Vitamin B6, Folate, Vitamin B12, Pantothenic Acid, Biotin, and Choline; National Academies Press: US Washington (DC), 1998.

(4) Fernandez-Mejia, C. Pharmacological effects of biotin. J. Nutr. Biochem. 2005, 16, 424-427.

(5) Koutsikos, D.; Agroyannis, B.; Tzanatos-Exarchou, H. Biotin for diabetic peripheral neuropathy. Biomed. Pharmacother. 1990, 44, 511-514.
(6) Shelley, W. B.; Shelley, E. D. Uncombable hair syndrome: observations on response to biotin and occurrence in siblings with ectodermal dysplasia. J. Am. Acad. Dermatol. 1985, 13, 97-102.

(7) Zempleni, J.; Hassan, Y. I.; Wijeratne, S. S. Biotin and biotinidase deficiency. Expert Rev. Endocrinol. Metab. 2008, 3, 715-724.

(8) US Food \& Drug Administration. Biotin (Vitamin B7): Safety Communication-May Interfere with Lab Tests. https://www.fda.gov/ $\mathrm{S}$ a f e t y / M e d W a t c h / S a f e ty I n f or m a t i o n / SafetyAlertsforHumanMedicalProducts/ucm586641.htm (accessed November, 2017).

(9) Samarasinghe, S.; Meah, F.; Singh, V.; Basit, A.; Emanuele, N.; Emanuele, M. A.; Mazhari, A.; Holmes, E. W. Biotin interference with routine clinical immunoassays: Understand the causes and mitigate the risks. Endocr. Pract. 2017, 23, 989-998.

(10) Green, N. M. Spectrophotometric determination of avidin and biotin. Methods in Enzymology; Academic Press, 1970; Vol. 18, p 418

(11) Marin, D.; Vera, J.; Serna, A. Polarographic reduction of biotin and its mechanisms. An. Quim. 1977, 73, 1243.

(12) Serna, A.; Vera, J.; Marin, D. Polarographic behaviour of biotin. J. Electroanal. Chem. Interfacial Electrochem. 1973, 45, 156-159.

(13) Lauw, S. J. L.; Ganguly, R.; Webster, R. D. The electrochemical reduction of biotin (vitamin $\mathrm{B} 7$ ) and conversion into its ester. Electrochim. Acta 2013, 114, 514-520.

(14) Opinion of the Scientific Committee on Food on the Tolerable Upper Intake Level of Biotin. https://ec.europa.eu/food/sites/food/ files/safety/docs/sci-com_scf_out106_en.pdf (expressed on 26 September, 2001).

(15) Mock, D. M. Biotin, 7th ed.; ILSI Nutrition Foundation: Washington, DC, 1996.

(16) Luong, J. H. T.; Male, K. B.; Glennon, J. D. Boron-doped diamond electrode: synthesis, characterization, functionalization and analytical applications. Analyst 2009, 134, 1965.

(17) Buzid, A.; Luong, J. H. T.; Reen, F. J.; O’Gara, F.; Glennon, J. D.; McGlacken, G. P. Rapid electrochemical detection of Pseudomonas aeruginosa signaling molecules by boron-doped diamond electrode. Methods Mol. Biol. 2018, 1673, 107-116.

(18) Buzid, A.; Reen, F. J.; Langsi, V. K.; Muimhneacháin, E. Ó.; O'Gara, F.; McGlacken, G. P.; Luong, J. H. T.; Glennon, J. D. Direct and rapid electrochemical detection of Pseudomonas aeruginosa quorum signaling molecules in bacterial cultures and cystic fibrosis sputum samples through cationic surfactant-assisted membrane disruption. ChemElectroChem 2017, 4, 533-541.

(19) Buzid, A.; Shang, F.; Reen, F. J.; Muimhneachain, E. O.; Clarke, S. L.; Zhou, L.; Luong, J. H. T.; O'Gara, F.; McGlacken, G. P.; Glennon, J. D. Molecular signature of Pseudomonas aeruginosa with simultaneous nanomolar detection of quorum sensing signaling molecules at a boron-doped diamond electrode. Sci. Rep. 2016, 6, 30001.

(20) Shang, F.; Muimhneacháin, E. Ó.; Jerry Reen, F.; Buzid, A.; O'Gara, F.; Luong, J. H. T.; Glennon, J. D.; McGlacken, G. P. One step preparation and electrochemical analysis of IQS, a cell-cell communication signal in the nosocomial pathogen Pseudomonas aeruginosa. Bioorg. Med. Chem. Lett. 2014, 24, 4703-4707.

(21) Buzid, A.; Muimhneacháin, E. Ó.; Reen, F. J.; Hayes, P. E.; Pardo, L. M.; Shang, F.; O’Gara, F.; Sperry, J.; Luong, J. H. T.; Glennon, J. D.; McGlacken, G. P. Synthesis and electrochemical detection of a thiazolyl-indole natural product isolated from the nosocomial pathogen Pseudomonas aeruginosa. Anal. Bioanal. Chem. 2016, 408, 6361-6367.

(22) Green, N. M. Avidin and streptavidin. Methods Enzymol. 1990, $184,51$.

(23) Groningsson, K.; Jansson, L. TLC determination of biotin in a lyophilized multivitamin preparation. J. Pharm. Sci. 1979, 68, 364366.

(24) Perez-Ruiz, T.; Martinez-Lozano, C.; Sanz, A.; Bravo, E. Electrophoretic behaviour of biotin and biocytin in capillary electrophoresis. Determination of biotin in pharmaceutical formulations. Chromatographia 2003, 58, 757. 
(25) Lahély, S.; Ndaw, S.; Arella, F.; Hasselmann, C. Determination of biotin in foods by high-performance liquid chromatography with post-column derivatization and fluorimetric detection. Food Chem. 1999, 65, 253-258.

(26) Przyjazny, A.; Hentz, N. G.; Bachas, L. G. Sensitive and selective liquid chromatographic postcolumn reaction detection system for biotin and biocytin using a homogeneous fluorophorelinked assay. J. Chromatogr. A 1993, 654, 79-86.

(27) Stein, J.; Hahn, A.; Lembcke, B.; Rehner, G. High-performance liquid chromatographic determination of biotin in biological materials after crown ether-catalyzed fluorescence derivatization with panacyl bromide. Anal. Biochem. 1992, 200, 89-94.

(28) http://www.chemicalbook.com/ChemicalProductProperty EN CB3271542.htm.

(29) Foley, J. K.; Korzeniewski, C.; Pons, S. Anodic and cathodic reactions in acetonitrile/tetra-n-butylammonium tetrafluoroborate: an electrochemical and infrared spectroelectrochemical study. Can. J. Chem. 1988, 66, 201-206.

(30) Sapountzi, F. M.; Gracia, J. M.; Weststrate, C. J.; Fredriksson, H. O. A.; Niemantsverdriet, J. W. Electrocatalysts for the generation of hydrogen, oxygen and synthesis gas. Prog. Energy Combust. Sci. 2017, 58, 1-35.

(31) Koch, V. R.; Miller, L. L.; Clark, D. B.; Fleischmann, M.; Joslin, T.; Pletcher, D. Anodic fluorination from fluoroborate electrolytes. J. Electroanal. Chem. Interfacial Electrochem. 1973, 43, 318-320.

(32) Pons, S.; Khoo, S. B. Reductions in aprotic media-I. Cathodic reduction limits at a platinum electrode in acetonitrile. Electrochim. Acta 1982, 27, 1161-1169.

(33) Geniès, L.; Faure, R.; Durand, R. Electrochemical reduction of oxygen on platinum nanoparticles in alkaline media. Electrochim. Acta 1998, 44, 1317-1327.

(34) Liu, W.; Samanta, S. K.; Smith, B. D.; Isaacs, L. Synthetic mimics of biotin/(strept)avidin. Chem. Soc. Rev. 2017, 46, 23912403.

(35) Melville, D. B. Biotin sulfoxide. J. Biol. Chem. 1954, 208, 495.

(36) Oh, K. An efficient epimerization of biotin sulfone derivatives to 2-epi-biotin analogs. Tetrahedron Lett. 2007, 48, 3685-3688.

(37) du Vigneaud, V.; Melville, D. B.; Folkers, K.; Wolf, D. E.; Mozingo, R.; Keresztesy, J. C.; Harris, S. A. The structure of biotin: a study of desthiobiotin. J. Biol. Chem. 1942, 146, 475.

(38) Hofmann, K.; Chen, C.; Bridgwater, A.; Axelrod, A. E. Furan and tetrahydrofuran derivatives. VII. The synthesis and biological activity of a number of oxybiotin homologs1. J. Biol. Chem. 1947, 69, 191-195.

(39) Ruis, H.; Brady, R. N.; McCormick, D. B.; Wright, L. D. Bacterial degradation of biotin. II. Catabolism of 14-C-homobiotin and 14-C-norbiotin. J. Biol. Chem. 1968, 243, 547.

(40) Pilgrim, F. J.; Axelrod, A. E.; Winnick, T.; Hofmann, K. The microbiological activity of an oxygen analog of biotin. Science 1945, $102,35-36$

(41) Spry, D. B.; Fayer, M. D. Proton transfer and proton concentrations in protonated Nafion fuel cell membranes. J. Phys. Chem. B 2009, 113, 10210-10221.

(42) Anantaraman, A. V.; Gardner, C. L. Studies on ion-exchange membranes.Part 1 . Effect of humidity on the conductivity of Nafion. J. Electroanal. Chem. 1996, 414, 115-120.

(43) Mock, W. L. Stable thiophene sulfoxides. J. Am. Chem. Soc. 1970, 92, 7610-7612.

(44) Yoshida, J.-I.; Sofuku, H.; Kawabata, N. Selective oxidation of sulfides to sulfoxides by a polymeric reagent electrochemically generated and recycled in situ. Bull. Chem. Soc. Jpn. 1983, 56, $1243-1244$.

(45) Vitamin H. (Biotin) ELISA. Catalog Number MO46019. http://mdbioproducts.com/sites/default/files/elisas/Vitamin_H_ (Biotin)_ELISA M046019.R03.pdf.

(46) Mock, D. M. Biotin analogs account for half of the total avidinbinding substances in human-plasma. Clin. Res. 1989, 37, A955.

(47) Fujiwara, M.; Ando, I.; Yagi, S.; Nishizawa, M.; Oguma, S.; Satoh, K.; Sato, H.; Imai, Y. Plasma levels of biotin metabolites are elevated in hemodialysis patients with cramps. Tohoku J. Exp. Med. 2016, 239, 263-267.

(48) https://en.wikipedia.org/wiki/List_of_human_blood components.

(49) Piketty, M.-L.; Prie, D.; Sedel, F.; Bernard, D.; Hercend, C.; Chanson, P.; Souberbielle, J.-C. High-dose biotin therapy leading to false biochemical endocrine profiles: validation of a simple method to overcome biotin interference. Clin. Chem. Lab. Med. 2017, 55, 817825 